\title{
Reconfigurable Optical Add-Drop Multiplexer Using Microring Resonators
}

\author{
Edwin Klein', Douwe Geuzebroek, Henry Kelderman, Gabriel Sengo, Nigel Baker ${ }^{2}$ and Alfred \\ Driessen
}

${ }^{1}$ Integrated Optical MicroSystems Group, MESA+ Research Institute, University of Twente, P.O.Box 217, 7500 AE Enschede, The Netherlands (email: e.j.klein@utwente.nl)

${ }^{2}$ Advanced Technology Networks Group, Nortel Networks, Harlow, Essex CM17 9NA, United Kingdom

\begin{abstract}
A reconfigurable four channel OADM based on vertically coupled thermally tunable $\mathrm{Si}_{3} \mathrm{~N}_{4} / \mathrm{SiO}_{2}$ microring resonators has a footprint of $0.25 \mathrm{~mm}^{2}$. Each MR can be tuned over $4.3 \mathrm{~nm}$ and has a bandwidth $>10$ GBit.
\end{abstract}

Keywords: optical networks, optical add-drop multiplexer, integrated optics, thermally tunable, microring resonator, $\mathrm{Si}_{3} \mathrm{~N}_{4}$

\section{Introduction}

As computers become more powerful and high-bandwidth applications, for instance for streaming media, become more prevalent, the demand for network bandwidth is growing rapidly. In order to satisfy this demand the deployment of optical networks is moving more and more towards the home-user's premises. Since deployment costs are a major issue in these access networks, low-cost optical filtering and switching functions need to be devised. Integrated optical Microring Resonators (MRs) are viable candidates for these functions as they combine a small footprint with a highly selective filter function $[1,2,3]$. Furthermore they can be readily combined into more complex structures such as a wavelength selective switch [4,5].

An important component in which the filter function and small size of MRs can be applied effectively is a WDM router. Figure 1 shows a possible 4-channel implementation of such a router [6]. This router consists of five 4-way Optical Add-Drop multiplexers (OADM). In this router the WDM input signal Iin is first separated into individual channels $\left(\lambda_{1} \ldots \lambda_{4}\right)$ by an OADM. Each of these channels is then guided into one of four additional OADMs. These OADMs can then add these channels to one of four output waveguides lout . $_{\text {. }}$

The OADMs can be implemented using AWGs or Mach-Zehnders. OADMs based on AWGs are generally large devices since AWGs can only split channels, and thus require additional components, while $\mathrm{MZ}$ based OADMs require multiple MZs for the selection of a single channel. An OADM based on MRs however offers several advantages over these conventional implementations. The use of MRs, which can have radii as small as $2 \mu \mathrm{m}$, allows

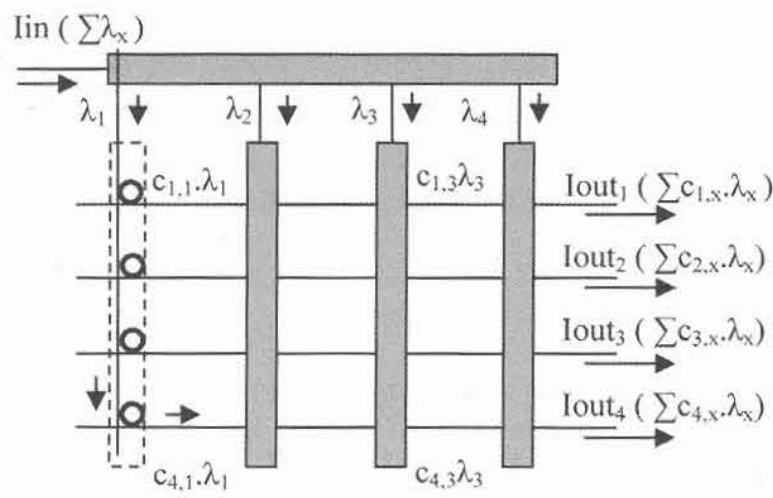

Figure 1: Schematic of a 4-channel WDM Router consisting of 5 connected OADM's for extremely small OADM implementations. In addition, a minimal component implementation of a 4-channel OADM based on MRs can already be realized with only four MRs, due to their highly selective filter characteristic. The first column of figure 1 shows such an implementation where each MR drops an incoming channel $\lambda_{x}$ to one of the outputs Iout $x_{x}$ when its resonance frequency corresponds to that of the incoming channel. In this paper the design, fabrication and measurements of a 4-channel thermally tunable MR based OADM are presented. 


\section{Design and fabrication}

The OADM was designed as shown in figure 2a. It consists of a central waveguide (Iin/Iout) and four Add/Drop waveguides. These waveguides are spaced at $250 \mu \mathrm{m}$ to allow for a standard fiber-array connection. The size of the OADM, $1.25 \times 0.2 \mathrm{~mm}^{2}$ is mainly determined by this spacing. A single MR is located at each intersection of central- and add-drop waveguides. The cross-grid waveguide approach [6,7], in which the two waveguides that couple to the MR cross each other, leads to some crosstalk but is also the most efficient geometry for the OADM. Each of the four MRs can be thermally tuned by a heater. The heater is omega shaped for high power efficiency. A 3D geometry and cross-section of a MR is shown in figure $2 \mathrm{~b}$. The MR has a radius of $50 \mu \mathrm{m}$, a height of $190 \mathrm{~nm}$ and a width of $2.5 \mu \mathrm{m}$, giving an Neff=1.517 (TE@1550 nm).The $50 \mu \mathrm{m}$ radius was chosen because it gives a FSR that is smaller than the thermal tuning range, allowing full FSR tuning. In addition, it allows for a MR that is nearly phase matched to the port waveguides. The MR is vertically coupled to these port waveguides which are $2 \mu \mathrm{m}$ wide, $140 \mathrm{~nm}$ high and have a Neff=1.505 (TE@1550 nm). Both the MR and the port waveguides are designed for TE operation.

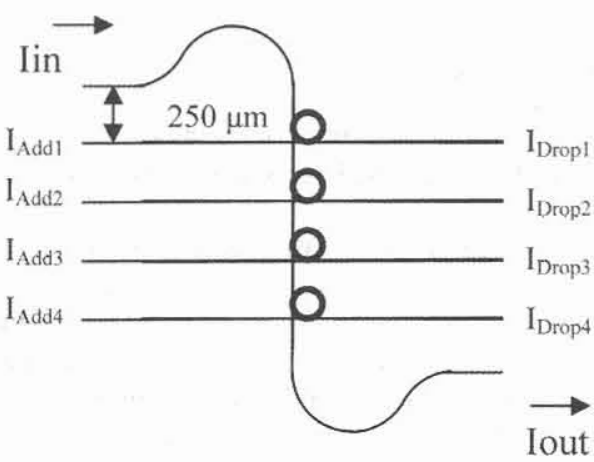

Figure 2a: Layout of the add-drop multiplexer

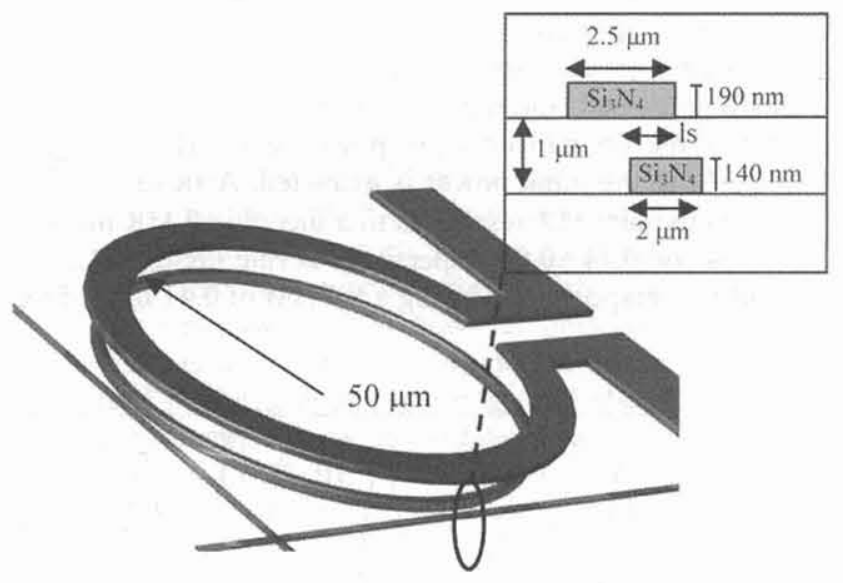

2b: MR top view and coupling region cross-section
The high aspect ratio in both the MR and port waveguides stems from lithographical limitations and the use of stoichiometric $\mathrm{Si}_{3} \mathrm{~N}_{4}$ ( $\left.\mathrm{n}=1.98 @ 1550 \mathrm{~nm}\right)$ which is limited to a maximum thickness of $340 \mathrm{~nm}$ to avoid material stress.

The OADM was fabricated by depositing $140 \mathrm{~nm}$ LPCVD $\mathrm{Si}_{3} \mathrm{~N}_{4}$ on top of $8 \mu \mathrm{m}$ thermally grown $\mathrm{SiO}_{2}[2,8]$. The $\mathrm{Si}_{3} \mathrm{~N}_{4}$ waveguides were then etched using reactive ion etching (RIE). A $1 \mu \mathrm{m}$ TEOS separation layer was applied next. The ring resonators were then defined by depositing $190 \mathrm{~nm} \mathrm{Si}_{3} \mathrm{~N}_{4}$ and RIE. A $4 \mu \mathrm{m}$ thick layer of PECVD $\mathrm{SiO}_{2}$ was deposited next. The device was then annealed at $1150^{\circ} \mathrm{C}$. Subsequently the $200 \mathrm{~nm}$ thick Chromium heaters were defined using lift-off.

Figure 3 shows a close up of a fabricated OADM. The central and add/drop waveguides are clearly discernable as well as the omega shaped heater elements on top of the four micro-ring resonators. The inset shows a pigtailed and packaged OADM chip. The OADM chip itself measures $10 \times 9 \mathrm{~mm}$ and contains a total of four OADMs of which only the center OADM is connected.

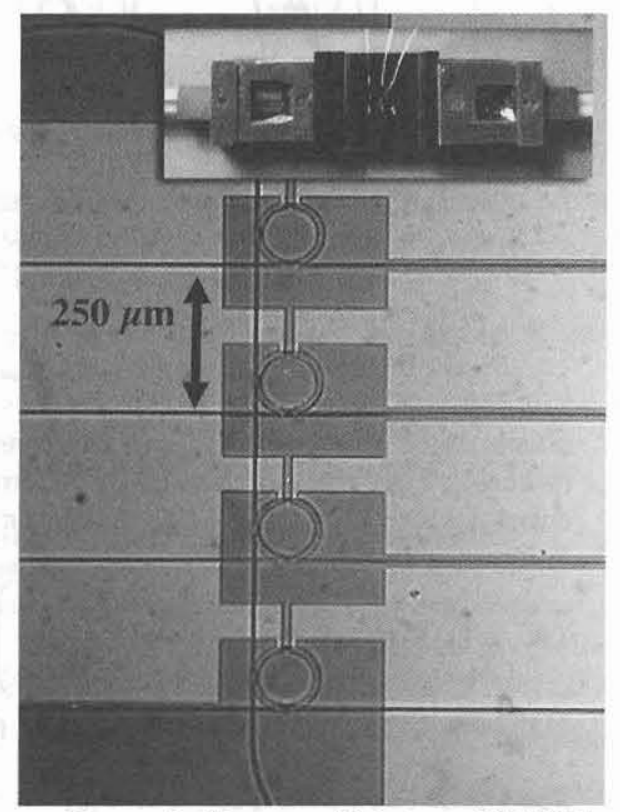

Figure 3: Closeup of fabricated OADM Inset: Pigtailed OADM 


\section{Measurement results and discussion}

The pigtailed OADM was measured using a broadband source and an optical spectrum analyzer with a resolution of $0.05 \mathrm{~nm}$. Figure 4 shows the normalized responses measured at Iout when the broadband source was connected to lin for two distinct configurations which were set by thermally tuning the MRs. In this setup the OADM now drops the channels present on lin to the desired drop ports $\mathrm{I}_{\mathrm{Drop} /}-\mathrm{I}_{\text {Drop4 }}$. These dropped channels are visible as MR through responses in Iout.

In the "4-channel configuration" a total heater power of $446 \mathrm{~mW}$ was applied to set the MR resonance frequencies on a $100 \mathrm{GHz}$ ITU Grid (spaced at $0.8 \mathrm{~nm}$ ). The minima of the individual $M R$ through responses are $\approx 12 \mathrm{~dB}$ below the normalized input power level. Thus $\approx 94 \%$ of the input power is extracted. A fit of

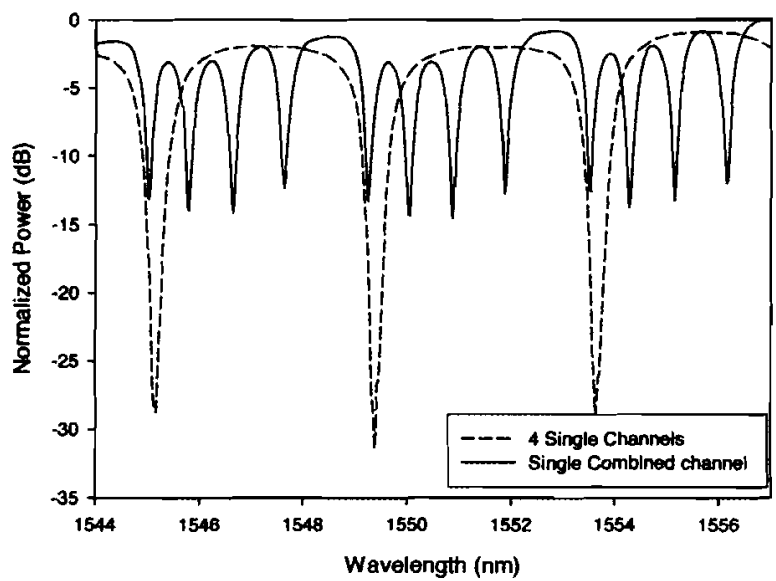

Figure 4: Iout response for a combined-channel and 4channel OADM configuration

the individual MR responses to a theoretical MR model showed amplitude coupling constants $\kappa_{1}$ and $\kappa_{2}$ of 0.56 \pm 0.04 and $0.44 \pm 0.04$ respectively at ring losses of $1.5 \pm 0.5 \mathrm{~dB} / \mathrm{cm}$. The measured FSR and Finesse were $4.18 \mathrm{~nm}$ and 10.3 respectively, giving a FWHM of $0.41 \mathrm{~nm}(=51 \mathrm{GHz})$.

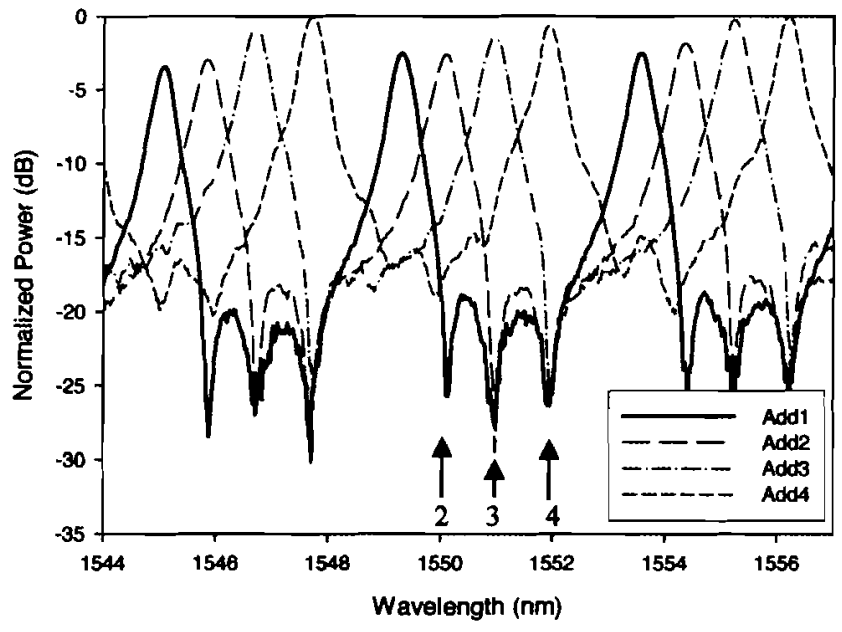

Figure 5: Add Responses for $I_{\text {AddI }} I_{\text {Add }}$ measured at lout.
The single channel configuration in figure 4 shows how the responses of the individual MRs could also be shifted to overlap each other. This configuration could be set while dissipating only $20 \mathrm{~mW}$ due to the fact that the untuned MRs already had nearly overlapping resonance frequencies, showing good fabrication reproducibility. The minimum of the combined through response is $30 \mathrm{~dB}(>99$ $\%$ extracted).

Figure 5 shows the normalized responses measured at Iout when the broadband source is connected to the different addports $\left(I_{\text {Add } 1}-I_{\text {Add4 }}\right)$. In this configuration the MRs select a channel from these ports and add it to lin.

The figure shows that minima of the individual $\mathrm{MR}$ drop responses are $\approx 17 \mathrm{~dB}$ below the normalized output power level $(\approx 89 \%$ of input power dropped). The

effects of the adjacent MRs on an add channel can also be observed, for instance in the add response of the first resonator. Here the three (indicated by arrows $2,3,4$ ) MRs that follow the first MR drop power from the main channel which shows up as dips in the Iout response. Using the parameters obtained from the fit to the responses in figure 4, the channel crosstalk [9]

$$
C T \equiv 10 \log \left(\frac{P_{\text {Drop }}\left(\lambda=\lambda_{c}+\Delta \lambda_{\text {ITU }}\right)}{P_{\text {Drop }}\left(\lambda=\lambda_{c}\right)}\right)=-10 \log \left(1+\frac{\sin ^{2}\left(\pi \cdot \Delta \lambda_{I T U} / F S R\right)}{\sin ^{2}(\pi / 2 F)}\right)
$$

of the device can be calculated to be $-11.7 \mathrm{~dB}$.

The OADM could be reconfigured in $<1 \mathrm{~ms}$ due to the fast thermal tuning response of the MRs [10]. Figure 6 shows that a single MR has a linear tuning response and has a maximum tuning range (without heater damage) 
of $\approx 4.3 \mathrm{~nm}$ at a power dissipation of $380 \mathrm{~mW}$, yielding a thermally induced wavelength shift of $11 \mathrm{pm} / \mathrm{mW}$ of dissipated heater power. The device shows no measurable thermal crosstalk due to the small heater area, wide $(\approx 150 \mu \mathrm{m})$ heater separation and the high thermal conductivity $(161 \mathrm{~W} / \mathrm{m} / \mathrm{K})$ of the silicon substrate.

Bandwidth measurements on the OADM have yet to be performed. Measurements carried out at Nortel on single MRs with the same geometry of the MRs used in the OADM have shown however that the bandwidth is $>10 \mathrm{GBit} / \mathrm{s}$ [5] while theory predicts a bandwidths $>20 \mathrm{GBit} / \mathrm{s}$.

\section{Conclusions}

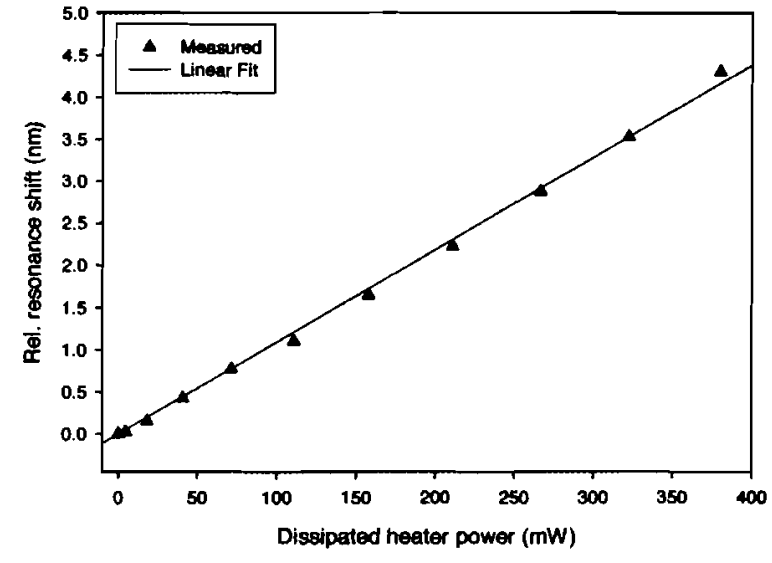

Figure 6: MR Tuning response

The feasibility of a reconfigurable add-drop multiplexer with thermally tunable $\mathrm{Si}_{3} \mathrm{~N}_{4} \mathrm{MRs}$ has been shown. Out of a large number of possible configurations a single- and a 4-channel configuration have been measured.

A change between the different OADM configurations could be made in $<1 \mathrm{~ms}$. Individual rings showed up to $12 \mathrm{~dB}$ extinction in the through port and $17 \mathrm{~dB}$ in the drop port. The rings have already been tested for bandwidth and showed good performance at $10 \mathrm{GBit} / \mathrm{s}$ [5] while even higher values are expected. The relatively low FSR of $4.18 \mathrm{~nm}$ of the MRs in the OADM will pose problems in network applications since multiple channels will be dropped within the $3^{\text {rd }}$ telecom window for which this OADM is intended. It is however possible to solve this issue by reducing the ring radius or utilizing the

Vernier effect by combining multiple MRs of different radii [11]. Using multiple rings will also further reduce the crosstalk which, with $-11.7 \mathrm{~dB}$ is still too high for practical applications. A cascade of two identical rings of the type used in the OADM for instance, will reduce the crosstalk to $-23 \mathrm{~dB}$.

\section{Acknowledgements}

The work has been carried out within the EC funded IST-project NAIS; Next Generation Advanced IntegratedOptic Subsystems, IST-2000-28018.

\section{References}

1) S.Suzuki et al., 'Integrated -optic ring resonators with two stacked layers of silica waveguide on Si', Photonic Technology Letters., vol. 4(11), pp. 1256-1258, 1992

2) F.C. Blom et al., 'Experimental study of integrated-optics microcavity resonators: Toward an all-optical switching device', Applied. Physics. Letters, vol. 71, pp. 747-749, 1997

3) B.Little et al., 'Microring resonator channel dropping filters', Journal of Lightwave Technology, vol. 15, pp. 9981005,1997

4) E.J.Klein et al., 'Wavelength Selective switch using thermally tunable microring resonators', Proceedings LEOS annual meeting, p. MM1, 2003

5) D.H.Geuzebroek et al., 'Compact Wavelength-Selective Switch for Gigabit Filtering in Access Networks', IEEE Photonic Technology Letters, Accepted for publication in February 2005.

6) B.Little et al., 'Microring resonator arrays for VLSI photonics', IEEE Photonic Technology Letters, vol, 12, pp. 323-325, 2000

7) Sai T.Chu et al., 'An Eight Channel Add-Drop Filter Using Vertically Coupled Microring Resonators over a Cross Grid', IEEE Photonic Technology Letters, Vol. 11. No. 6, pp. 691-693, June 1999

8) K.Worhoff et al., 'Silicon oxinitride - a versatile material for integrated optics applications', J. Electrochemical Society, vol. 149(8), pp. F85-F91, 2002

9) D.Klunder et al., 'Vertically and laterally waveguide-coupled cylindrical microresonators in $\mathrm{Si}_{3} \mathrm{~N}_{4}$ on $\mathrm{SiO}_{2}$ technology', Applied Physics B, vol. 73, pp. 603-708, 2001

10) R. Meijerink et al., 'Thermally tunable, wide FSR switch based on micro-ring resonators', Proceedings Symposium IEEE/LEOS Benelux, pp. 155-158, 2002

11) R.Grover et al. 'Parallel-Cascaded Semiconductor Microring Resonators for High-Order and Wide-FSR Filters', Journal of Lightwave Technology, vol. 14, no. 10, pp. 2260-2267, 1996 